$16^{\text {th }}$ International Conference on

AEROSPACE SCIENCES \& AVIATION TECHNOLOGY,

ASAT - 16 - May 26 - 28, 2015, E-Mail: asat@mtc.edu.eg

Military Technical College, Kobry Elkobbah, Cairo, Egypt

Tel : +(202) 24025292 - 24036138, Fax: +(202) 22621908

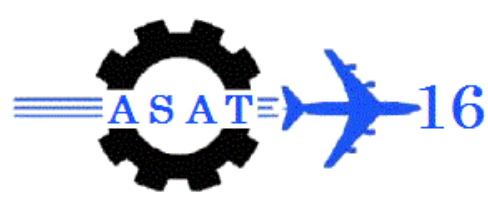

\title{
Surrogate Representation for the Aerodynamic Coefficients of Supersonic Airfoils
}

\author{
M. Y. M. Ahmed*
}

\begin{abstract}
Generally, physical phenomena are represented in the form of explicit mathematical expressions that stem from basic governing equations. However, in many cases, having these expressions may not be achieved and a considerable part of our knowledge relies on abstract numbers produced via lab experiments or computer simulations. In the absence of explicit relations for the variables of the physical phenomena, surrogates emerge as a reliable alternative. Surrogates have evolved in the recent years and gained the attention of researchers and, in the field of aerodynamics; surrogates have been widely used in multiple applications. In the present paper, surrogates are implemented in a new application namely, the relation between the design of a 10\% diamond airfoil and its aerodynamic coefficients. Kriging interpolation and polynomial regression surrogates are constructed based on data taken from CFD simulations of the turbulent, Mach 2, zero-incidence flow about 104 airfoil samples representing almost all possible designs of that airfoil.

The constructed surrogates are proved to incorporate the CFD data with a high accuracy. They also yield useful visual representations of the physical phenomena in hand and generate simple mathematical expressions for these phenomena as well.
\end{abstract}

Keywords: Supersonic airfoils, aerodynamic coefficients, surrogates

\section{Introduction}

In contrast to subsonic and transonic airfoils, the supersonic airfoils are characterized by a sharp leading edge. This key design feature is intended to generate a straight, attached shock wave ahead of the airfoil much weaker than the detached bow shock wave generated ahead of airfoils with blunt leading edges. Downstream of the leading edge, the flow over the airfoil subsequently expands as it passes through a series of expansion waves. The typical flow pattern around a symmetric diamond-shaped airfoil at zero incidence is illustrated in Fig. 1.

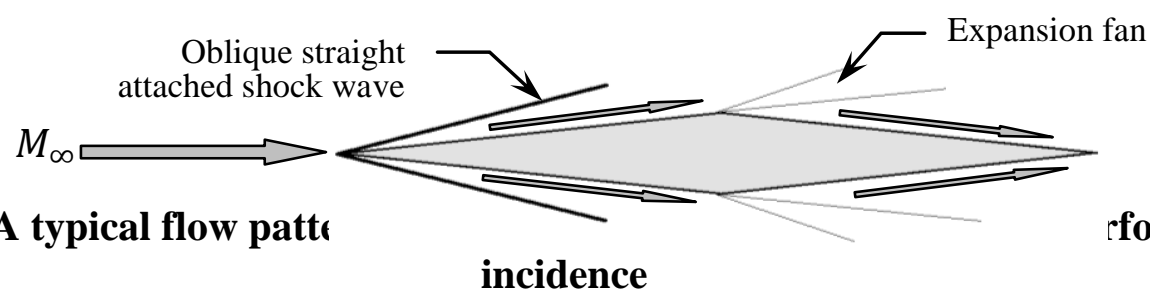

rfoil at zero

Figure 1. A typical flow patt

incidence

The flow pattern becomes more sophisticated as the complexity of the airfoil design increases, in cases of non-symmetric airfoils and/or at incidence. In all cases, the local flow direction is parallel to the airfoil surface whereas the local flow properties depend on the strengths of the shock waves and expansion fans. As a consequence, at given freestream Mach number, $M_{\infty}$, and incidence angle, the aerodynamic coefficients of the airfoil are solely dictated

by its design.

\footnotetext{
"Egyptian Armed Forces, Egypt.
} 
The aerodynamic coefficients of supersonic airfoils can be estimated using two approaches namely, the exact shock-expansion theory and the linearized supersonic flow theory. The shock-expansion theory [1] is an exact theory that is derived from the fundamental governing equations of inviscid compressible flow. It is based on a stepwise treatment of the supersonic flow over a body of arbitrary shape as a set of shock waves and expansion fans. By treating each of them separately, the flow properties over the entire airfoil can be estimated for known freestream flow properties. In contrast, the linearized supersonic flow theory [2] stems from the linear theory dealing with bodies that generate small disturbances in the flow. The linearized theory provides very simple closed-form equations that are used to estimate the flow properties over the entire airfoil.

However, both the exact shock-expansion and the linearized theories assume that the flow is potential. Hence, they can't predict the skin friction drag on the airfoil. More importantly, they fail to deal with situations where the flow separates on the airfoil surface. The only way to account for such aspects is to simulate the flow around the airfoil using computational fluid dynamics (CFD) techniques. In CFD simulation, the governing equations (depending on the nature and regime of flow) are solved numerically in a discretized domain around the airfoil to yield the flow properties over the airfoil surfaces. Nonetheless, CFD simulations are more time-consuming and resource-demanding when compared to both theories since they involve the activities of domain generation, discretization, and inspection, model selection and validation, and post-processing. In fact, CFD simulations may seem to be impractically expensive as far as simple aerodynamic problems are concerned.

Surrogates (also known as surrogate models, response surface models, metamodels, or models of the models) have emerged as a compromise solution in the fields involving expensive CFD simulations. The concept of surrogates is simple. In the absence of explicit mathematical expression of a given physical phenomenon, surrogates provide an alternative representation of the functional relations between independent and dependent parameters of that phenomenon. In practice, data (representing the physical phenomenon) are manipulated by some means to yield explicit mathematical expressions that can be used in lieu of the underlying physical relations. These data represent the inputs-output functional relations and, hence, inherently convey the underlying physical phenomena. These data, known as the training samples, can be originally extracted from diverse sources such as experimental measurements and samples, CFD simulations, etc..

In the literature, there exist a considerable number of surrogates however, they can be classified into two broad categories depending on the way they incorporate the data used in their construction. Surrogates can have either regression or interpolation structure. In the regression structure, the surrogate model is simply fitted among the available data whereas in the interpolation structure, the surrogate model actually passes through all the available data. In the present study, two well-known surrogates, each representing a category, are implemented.

Polynomial regression surrogate is a parametric regression surrogate. It was originally tailored for physical experiments in which the output is characterized by random errors and later introduced to computer-based experiments by Montgomery and Evans [3] to account for the random numerical errors. In this method, the surrogate is represented by a polynomial plus an error:

$\hat{y}=y+\varepsilon$

where $\varepsilon$ represents the random error. The approximation polynomial can have any order however, in most engineering applications, researchers used polynomials of the first order (linear):

$\hat{y}=\beta_{o}+\sum_{i=1}^{k} \beta_{i} x_{i}$ 
and second order (quadratic):

$\hat{y}=\beta_{o}+\sum_{i=1}^{k} \beta_{i} x_{i}+\sum_{i=1}^{k} \sum_{j \geq i}^{k} \beta_{i j} x_{i} x_{j}$

$\beta_{o}$ and $\beta_{i j},(i \neq j)$ are called the intercept and the interaction coefficients, respectively. The polynomial coefficients are estimated via minimizing the sum of squares of the deviations of predicted values from the original data.

Polynomial regression metamodels have been implemented in a wide range of aerodynamic applications including helicopter rotor blades [4], launch vehicle wing [5], NASA rotor67 [6], high-speed civil transport [7], flying wings [8], transonic airfoils [9], and predicting the aerodynamic coefficients of cranked wings [10].

Kriging is a non-parametric interpolating surrogate. It was originally introduced by D. G. Krige (and was named after him) in the field of geology to predict the concentration patterns of mineral resources in a field using samples [11]. Later, Kriging was developed and made popular in computer-based experiments by Sacks et al. [12]. The mathematical expression of Kriging metamodel comprises two parts, a mean (trend) function and localized deviations (pulls) from the mean at the training samples

$$
\hat{y}(x)=f(x)+z(x)
$$

The mean function is a linear regression (of arbitrary order) of the training samples representing the trend of the response based on the available data. In common engineering problems, the mean takes the form of a constant or a first-order polynomial. The second term in Eqn. (2.10) has a form of a Gaussian stochastic function with a correlation structure based on the generalized distances among training samples; the spatial correlation function, $s c f(d)$ of the Euclidian distance among samples, $d$. Generally, $s c f(d)$ can have many forms; typically linear, cubic, or exponential. The exponential correlation function has the most flexible structure that can handle phenomena with variable complexity. It is expressed as:

$$
s c f(d)=\exp \left(-\theta \times d^{p}\right) \text {. }
$$

The value of power, $p$, varies from 1 to 2 . The spatial correlation function is referred to as exponential, general exponential, or Gaussian if the value of the power is $p=1$, $1<p<2$, and $p=2$, respectively.

The most characteristic feature of the Kriging metamodel is the large number of undetermined parameters namely, the mean, the model variance, and the parameters $p$ and $\theta$ , the latter can have the same value for all parameters or different values for different parameters [13]. They should be carefully "tuned" to ensure the "best fit" of the model to the available training samples.

In the literature, Kriging surrogate was widely used in many aerodynamic applications including transonic airfoils [14], multi-element airfoils [15], flow control jets [16], engine nacelle [17], spiked hypersonic vehicles [18], HSCT and oblique wing body (OWB) designs [19], and supersonic projectiles [20].

The objective of the present study is to examine the concept of representing the aerodynamic coefficients of a supersonic diamond airfoil using surrogate models. Focus is made on lift and drag coefficients at zero incidence for $10 \%$ thick diamond airfoils of different designs. The paper is organized as follows. The case study and methodology are explained in detail in the next section. Presentation and discussion of the main results follow and the paper finalizes with the main conclusions. 


\section{II.1. Case study}

\section{Case study and Methodology}

A non-symmetric diamond-shaped airfoil of a unit chord, $c=1$, representing the section of a unit-span wing, is considered. The maximum thickness of both the upper and lower surfaces are fixed and equal to $5 \%$ of the chord whereas the locations of the maximum thickness per chord on both surfaces, $l_{U}$ and $l_{L}$, are variable and different, Fig. 2.

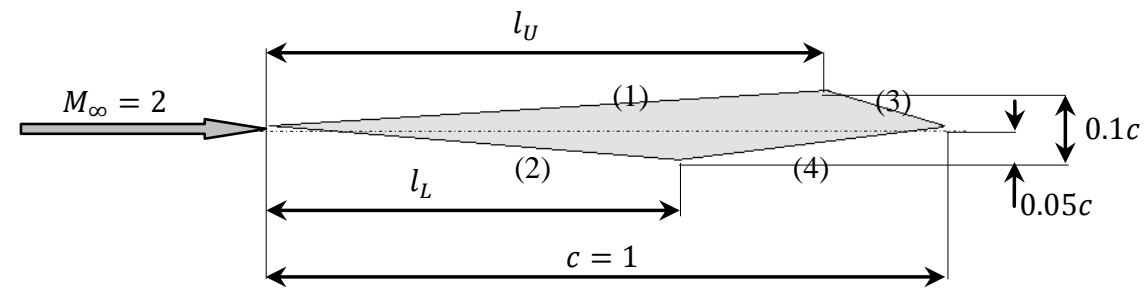

Figure 2. Geometry of the test case

The airfoil is exposed to a supersonic freestream of Mach, $M_{\infty}=2$, at zero incidence. The values of $l_{U}$ and $l_{L}$ should be high enough to ensure that an attached shock wave is generated on surfaces (1) and (2), respectively. For the value of the freestream Mach number considered here and due to similarity, both $l_{U}$ and $l_{L}$ should be at least 0.137 of the airfoil chord length.

\section{II.2. Design of experiments}

By varying the values of $l_{U}$ and $l_{L}$, different designs can be attained. Consequently, the design space in concern is two-dimensional with $l_{U}$ and $l_{L}$ as the coordinates. They are varied independently from their corresponding minimum values up to $c$ with 100 intermediate steps. A full factorial sampling would generate 10000 distinct designs that would be extremely computationally expensive. Thus, Latin hypercube sampling (LHS) [21] is adopted. LHS has the advantage of producing a space-filling sampling with a reduced number of samples. The two design parameters are first normalized to the range $[0,1]$ such that the value of 0 refers to the minimum allowable value of $l_{U}$ and $l_{L}$ mentioned above namely, 0.137 of the airfoil chord. Then, 100 values for each design parameter are selected such that none of the samples have the same values for $l_{U}$ and $l_{L}$. Four samples are manually added at the "corners" of the design space to ensure that the extreme designs are included in the study. The locations of the 104 samples in the 2D design space are shown in Fig. 3.

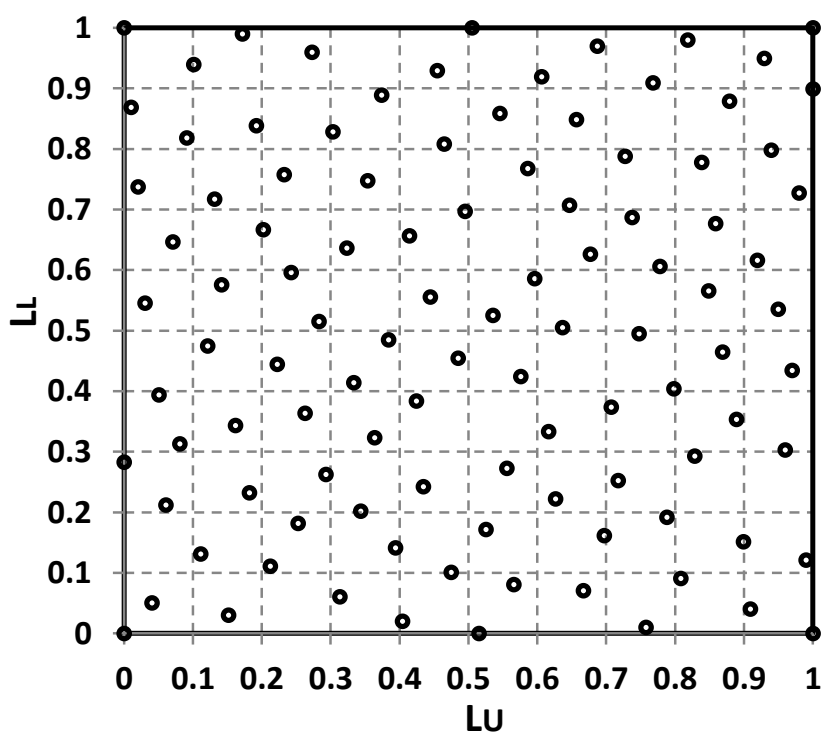

Figure 3. Design of the CFD experiments 


\section{II.3. Setting the CFD simulations}

\section{Computational domain and boundary conditions}

For each airfoil design, a 2D computational domain is constructed; a sample is shown in Fig. 4a. The domain shape and extents are designed so as to incorporate the shock waves and expansion fans that are generated on the airfoil and to make the computational grid more aligned with these features.

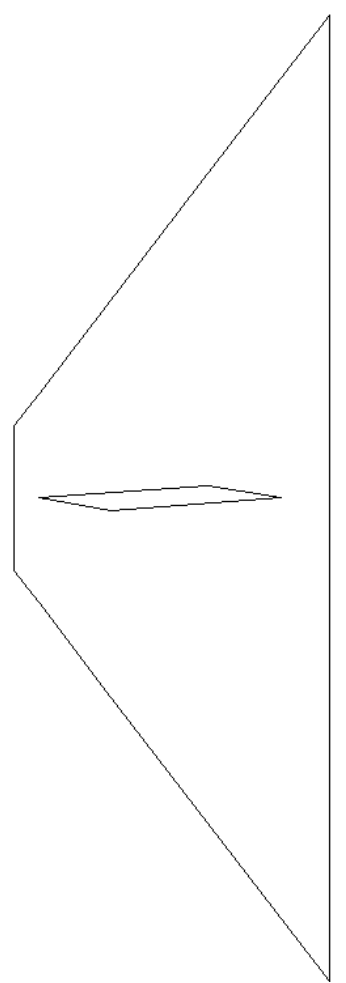

(a)

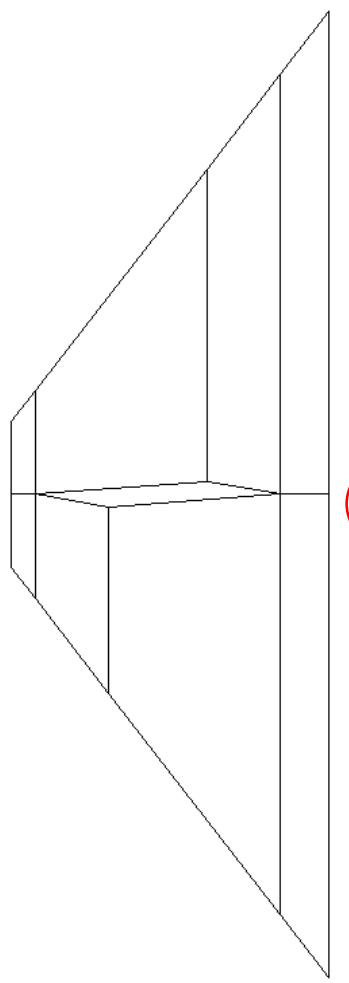

(b)

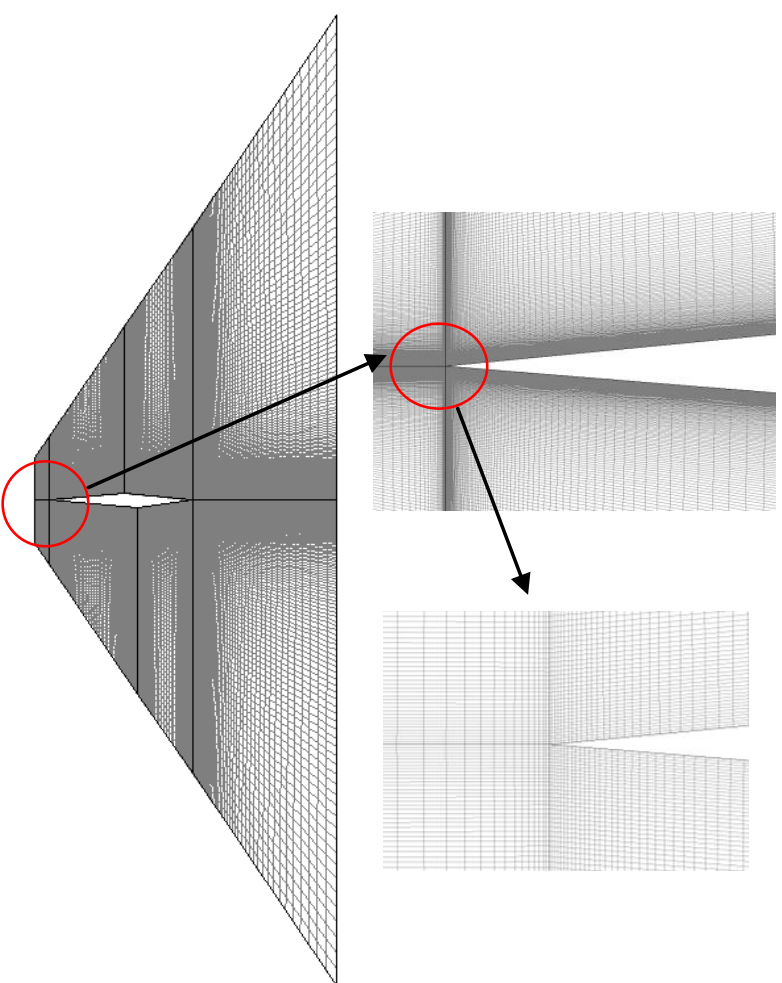

(c)

Figure 4 Details of the computational domain around a sample airfoil

Definitions of the boundary conditions are as follows. The upstream boundary is set as pressure farfield where the flow pressure, static temperature, Mach number, and direction are defined. The downstream end of the domain is set as pressure outlet where the flow total temperature is defined. The computational domain is then divided into multiple blocks, Fig. $4 \mathrm{~b}$, that are discretized using a structured grid, Fig. 4c. The grid resolution is increased at the leading and trailing edges of the airfoil and the junctions of airfoil surfaces as illustrated in the close-ups in Fig. 4c.

\section{Grid sensitivity check}

Three grids of different resolutions are constructed on a sample airfoil to make sure that the simulation results are independent of the spatial discretization. $l_{U}$ and $l_{L}$ of the tested airfoil are 0.7 and 0.3 of the chord, respectively. Table 1 summarizes the metrics of the grids and the corresponding values of total drag coefficient.

Table 1 Results of the grid sensitivity check

\begin{tabular}{rccc}
\hline \hline & \multicolumn{2}{c}{ Grid metrics } & \\
& Cell height at wall & Cell count & Drag coefficient \\
\hline Coarse grid & 0.001 & 28000 & 0.0278 \\
Medium grid & 0.0001 & 28000 & 0.0277 \\
Fine grid & 0.0001 & 53000 & 0.02778 \\
\hline \hline
\end{tabular}

The medium and fine grids yield almost the same value of drag coefficient; the difference is only 0.8 drag counts. The medium grid would indeed be less computationally 
expensive however, the fine grid metrics were adopted in the simulations since the simulation budget was affordable.

\section{CFD code validation}

To validate the CFD code, one case from the theoretical investigation of Ivey et al. [1] (based on the exact shock-expansion theory) is reproduced numerically. The validation case is a symmetric diamond-shaped airfoil of equal leading and trailing-edge angles of $2^{o}$ placed at $3^{o}$ incidence in a Mach 4 freestream. The metrics of the fine grid were utilized in the simulations. Table 2 holds a comparison between the theoretical and calculated values of local pressure coefficients and Mach numbers on the four surfaces of the airfoil. The accuracy of calculations of the code is assumed acceptable. CFD simulations are conducted for each of 104 airfoil designs using the validated CFD model based on the validated grid.

Table 2 Validation results for the calculation code

\begin{tabular}{rcccc}
\hline \hline & \multicolumn{2}{c}{ Local pressure coefficient } & \multicolumn{2}{c}{ Local Mach number } \\
& Theory [1] & CFD & Theory [1] & CFD \\
\hline Forward windward & 0.0416 & 0.042 & 3.7 & 3.69 \\
Rearward windward & 0.0188 & 0.019 & 3.84 & 3.82 \\
Forward leeward & -0.0169 & -0.0163 & 4.16 & 4.142 \\
Rearward leeward & -0.0308 & -0.0306 & 4.33 & 4.299 \\
\hline \hline
\end{tabular}

\section{II.4. Surrogate models}

Upon completion of all CFD simulation cases, the aerodynamic coefficients for all airfoil designs are estimated. Based on these high-fidelity values, surrogates are constructed for each aerodynamic performance criteria namely, lift and drag coefficients.

Six types of Kriging surrogates as well as two types of polynomial regression surrogates are examined in the present work. These surrogates are listed in the Table below. for easier referencing, each surrogate type is given a special designation that is used in the remainder of the paper.

Table 3 Description of surrogates

\begin{tabular}{|c|c|c|}
\hline $\begin{array}{r}\text { Surrogate } \\
\text { Designation } \\
\end{array}$ & Description & Mathematical representation \\
\hline GEX0 & $\begin{array}{lc}\text { Kriging } & \text { surrogate with a } \\
\text { constant } & \text { mean and a } \\
\text { general } & \text { exponential } \\
& \text { correlation }\end{array}$ & $\begin{array}{r}f\left(l_{U}, l_{L}\right)=\beta_{o} \\
s c f(d)=\exp \left(-\theta \times d^{p}\right), 1<p<2\end{array}$ \\
\hline GEX1 & $\begin{array}{l}\text { Kriging surrogate with a } \\
\text { first-order mean and a } \\
\text { general }\end{array}$ & $\begin{array}{r}f\left(l_{U}, l_{L}\right)=\beta_{o}+\beta_{1} l_{U}+\beta_{2} l_{L} \\
\operatorname{scf}(d)=\exp \left(-\theta \times d^{p}\right), 1<p<2\end{array}$ \\
\hline EX0 & $\begin{array}{l}\text { Kriging surrogate with a } \\
\text { constant mean and an } \\
\text { exponential correlation }\end{array}$ & $\begin{array}{r}f\left(l_{U}, l_{L}\right)=\beta_{o} \\
s c f(d)=\exp (-\theta \times d)\end{array}$ \\
\hline EX1 & $\begin{array}{l}\text { Kriging surrogate with a } \\
\text { first-order mean and an } \\
\text { exponential correlation }\end{array}$ & $\begin{array}{r}f\left(l_{U}, l_{L}\right)=\beta_{o}+\beta_{1} l_{U}+\beta_{2} l_{L} \\
\operatorname{scf}(d)=\exp (-\theta \times d)\end{array}$ \\
\hline GU0 & $\begin{array}{l}\text { Kriging surrogate with a } \\
\text { constant mean and a } \\
\end{array}$ & $f\left(l_{U}, l_{L}\right)=\beta_{o}$ \\
\hline
\end{tabular}




\begin{tabular}{rrr}
\hline \hline Gaussian correlation & $s c f(d)=\exp \left(-\theta \times d^{2}\right)$ \\
\hline GU1 & $\begin{array}{l}\text { Kriging surrogate with a } \\
\text { first-order mean and a } \\
\text { Gaussian correlation }\end{array}$ & $f\left(l_{U}, l_{L}\right)=\beta_{o}+\beta_{1} l_{U}+\beta_{2} l_{L}$ \\
PR1 & $\begin{array}{l}\text { Polynomial regression } \\
\text { surrogate of the first order }\end{array}$ & $s c f(d)=\exp \left(-\theta \times d^{2}\right)$ \\
\hline PR2 & $\begin{array}{l}\text { Polynomial regression } \\
\text { surrogate of the second } \\
\text { order }\end{array}$ & $\hat{y}=\beta_{o}+\beta_{1} l_{U}+\beta_{1} l_{U}+\beta_{2} l_{L}+\beta_{2} l_{U} l_{L}+\beta_{4} l_{U}^{2}+\beta_{5} l_{L}^{2}$ \\
\hline \hline
\end{tabular}

\section{III.1. The typical flowfield structures}

\section{Results and Discussion}

Indeed, the flowfield features would change from each of the 104 airfoil design to another depending on values of $l_{U}$ and $l_{L}$. Since this is not the scope of the present study, focus is made on four airfoil designs that yield the extreme values for both lift and drag coefficient. The flowfield features around these designs are illustrated in Figure 5 where the contours of the flow pressure coefficients are plotted.

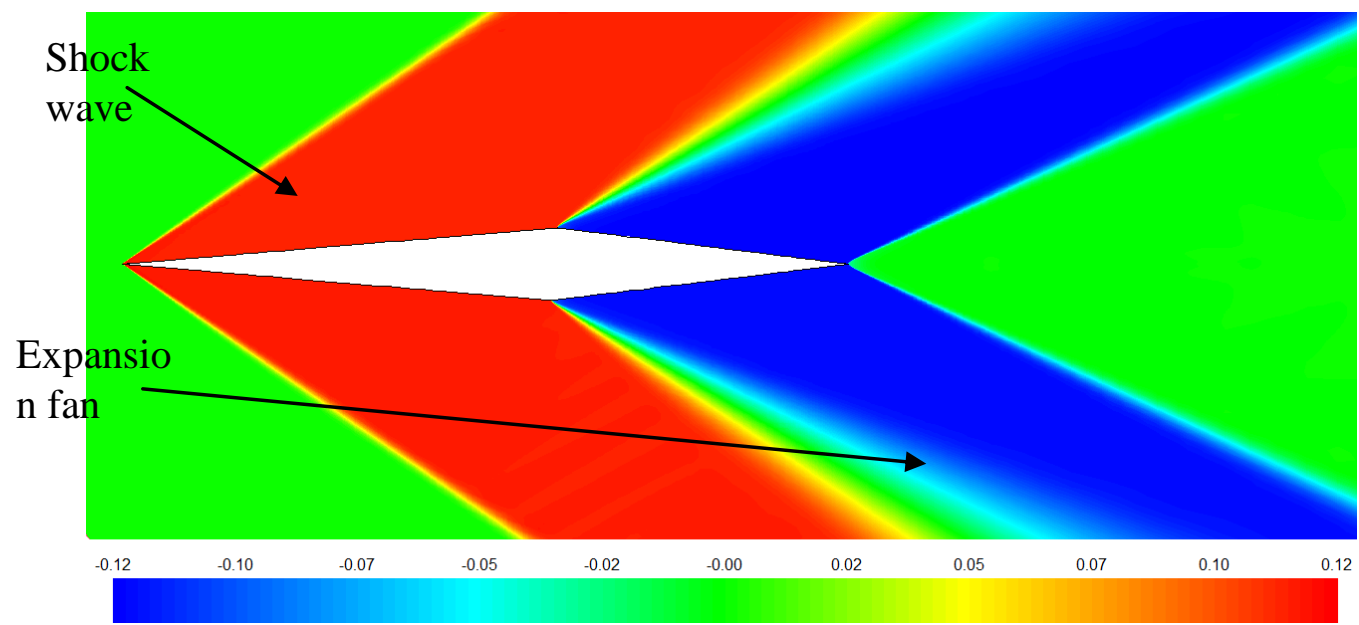

(a) Model with minimum drag coefficient

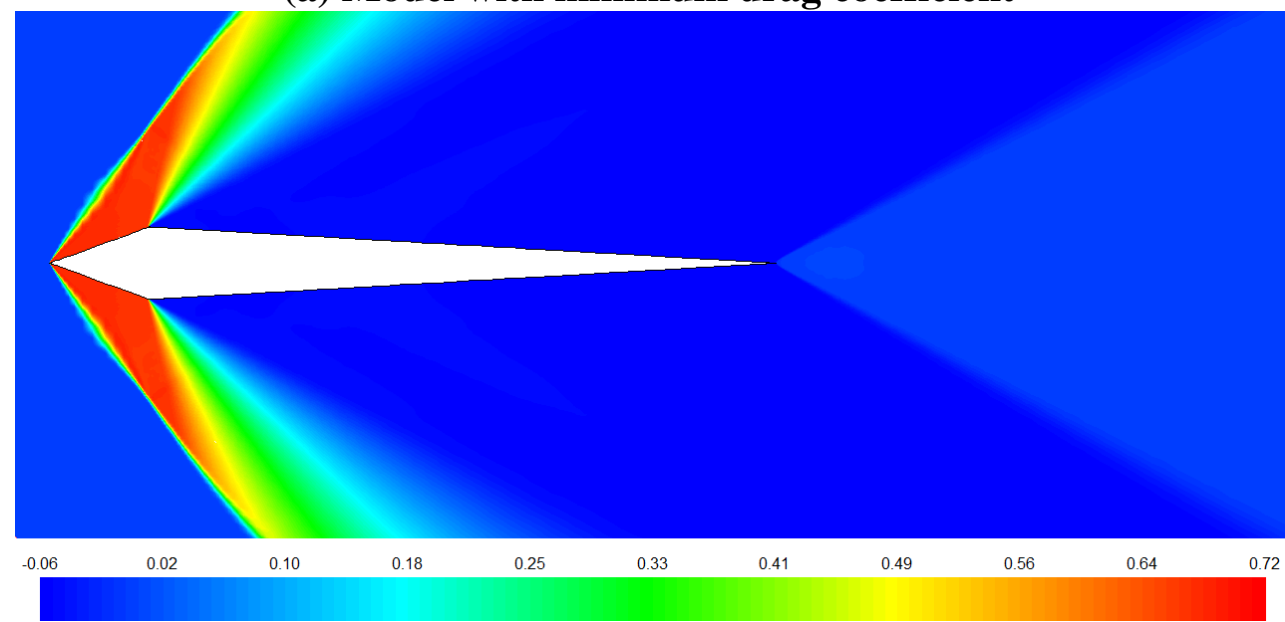

(b) Model with maximum drag coefficient 

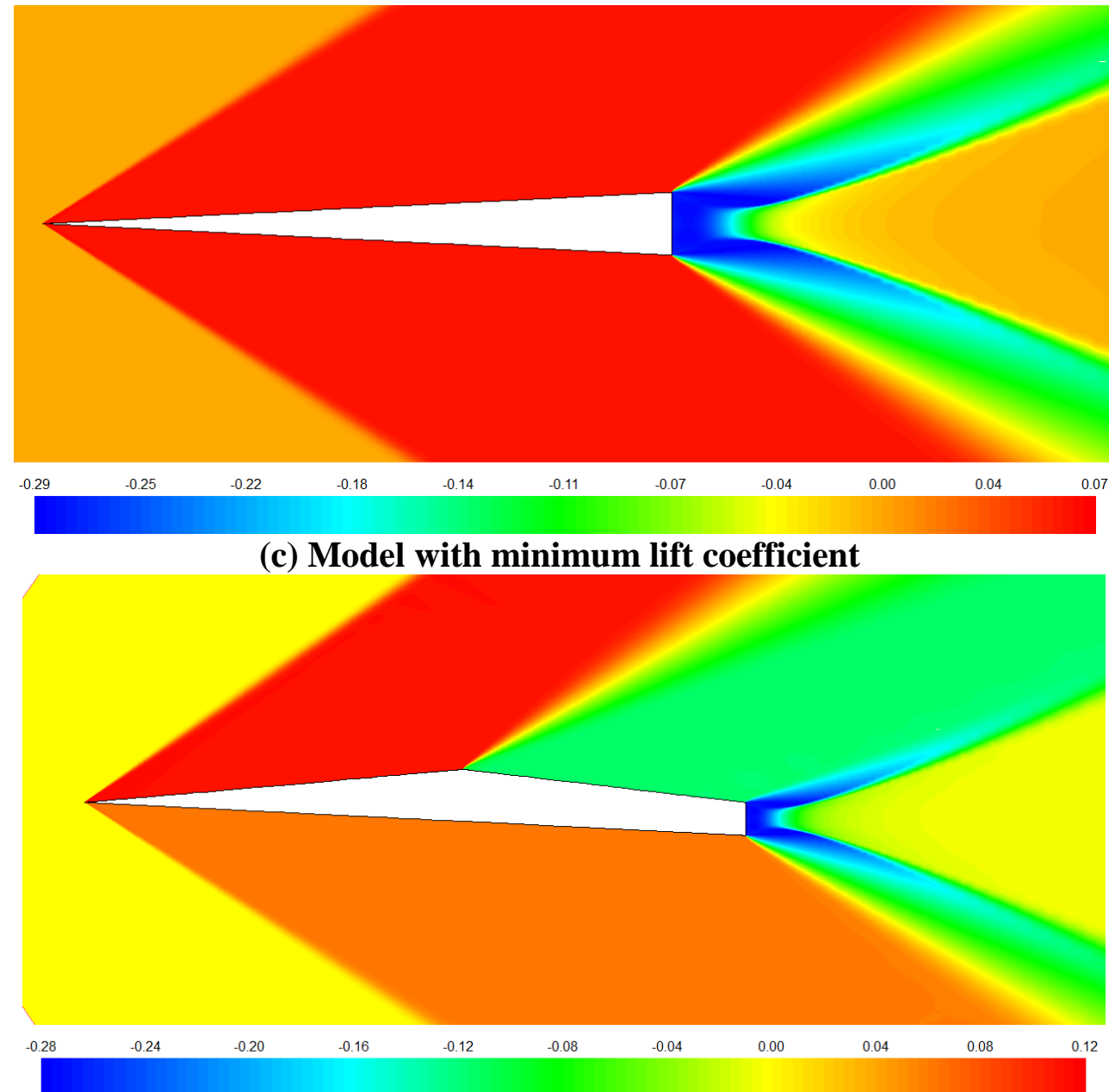

(d) Model with maximum lift coefficient

Figure 5 Flow pressure contours around models with extreme lift and drag coefficients

Indeed, the controlling flow features are the shock waves and expansion fans. The strength of these two features dictate the local pressure values over the airfoil surfaces and is, in the same time, governed by the surfaces' dimensions.

For the freestream conditions investigated here, the airfoil that yields the minimum drag is characterized by a symmetric design; $l_{U}=l_{L} \approx 0.6$, Fig. 5a. This design implies that the shock wave and expansion fan strength on both upper and lower surfaces are such that the local pressure coefficients on the forward surfaces are equal (and opposite in sign) to those on the rearward surfaces. Overall, the drag coefficient is minimized and is owed mainly to the skin friction drag on the airfoil surfaces. In contrast, drag attains a maximum value for the airfoil at which the shock waves ahead of the leading edge are as strong as possible. This occurs for the design with $l_{U}=l_{L}=l_{\min }=0.137$, Fig. $5 \mathrm{~b}$. Since the forward surfaces are the shortest, the subsequent expansion ahead of the rearward surfaces is strong, too. The overall effect is that the drag is maximized.

At zero incidence, lift is minimized for all symmetric airfoil designs such as those shown in Fig. 5a to c. Symmetry of airfoil designs (about the chord line) yields identical shock waves and expansion fans of the same respective strengths on the upper and lower surfaces of the airfoil. The resulting similarity of the local floe pressure values produces no lift on the airfoil. Finally, the airfoil in Fig. 5d produces the highest lift among the 104 samples investigated. Despite that pressure over the upper forward surface is higher than that over the lower, the combined effect of pressure along with surfaces dimensions and inclinations yields the maximum lift coefficient. 


\section{III.2. Surrogate assessment}

A major precaution of using surrogates is that they introduce a degree of uncertainty to the predicted response [22]. Hence, it is crucial to examine their quality before using them as an alternative model. Evaluating the residual errors at the training samples can be useful. However, this can only be applied to regression surrogates; interpolation surrogates would give zero residual error at the training samples. In addition, it is more appropriate to assess the accuracy of prediction or the generalization error estimation of the surrogate. Generally, this is done by comparing the surrogate responses at new (untried) samples, the test samples, with their high fidelity model counterparts. The error at these new samples is measured by a number of error metrics such as the mean square error, the root mean square error, the mean absolute error, or the maximum absolute error. To select the test samples, there are two approaches namely, split sample and cross-validation [23].

In the split sample approach, the available samples are split into two sets, training and test sample sets. The former is exclusively used to construct the surrogate whereas the latter is exclusively used to assess its accuracy. The error at the test samples is measured by a number of error metrics such as the root mean square error (RMSE), the maximum absolute error (MAE), and the maximum error (MAXE). On the other hand, in the cross-validation approach, all the available samples are used in constructing the surrogate. Next, the samples are divided into $k$ subsets of roughly equal sizes ( $k$-fold cross-validation). Then, the surrogate is constructed $k$ times. In each time, one of the $k$ subsets is omitted from the training process and is used as test set (this approach is also known as leave- $k$-out crossvalidation. In the cross-validation approach, the surrogate accuracy is measured by the mean square error (MSE).

In the present study, both surrogate assessment techniques are implemented. In the split-sample techniques, the original set containing the 104 airfoil designs is split into two groups; the training set containing 84 samples, and the test set containing 20 samples. The locations for samples in the two sets in the design spaces are illustrated in Fig. 6 below.

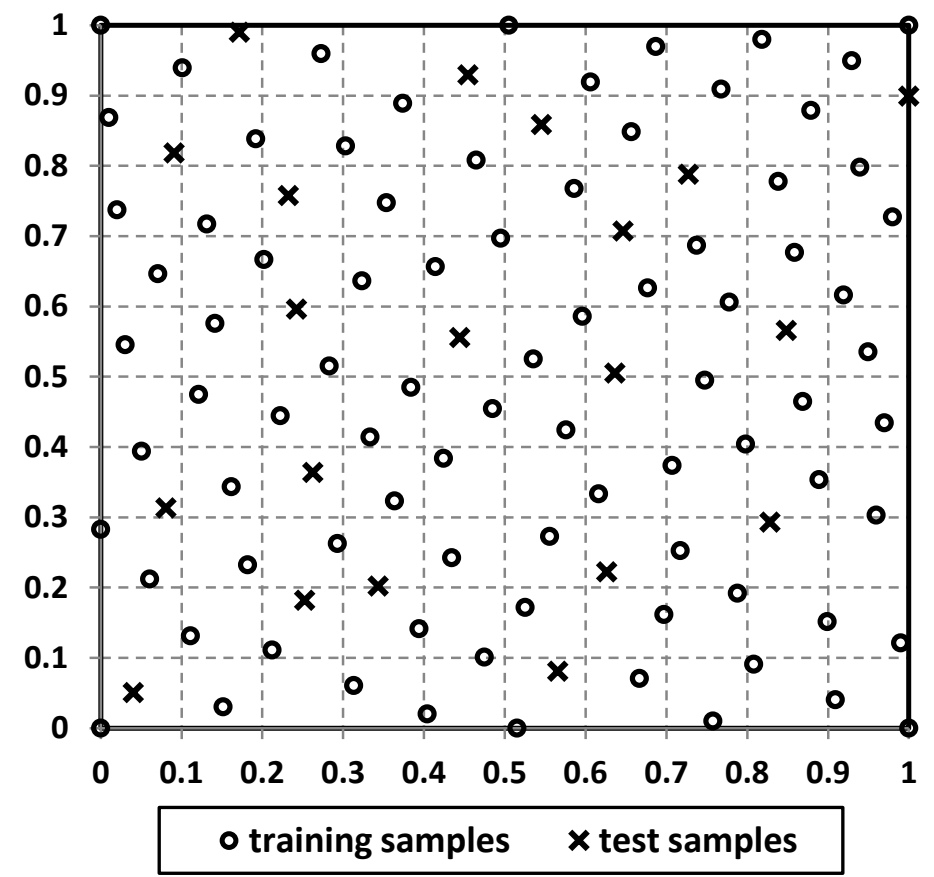

Figure 6 Locations of training and test samples in the design space

The results of surrogate assessment are summarized in Fig.7a and b for lift and drag coefficients, respectively. It can be clearly inferred that the exponential Kriging surrogate with a first-order mean (EX1) yields the most accurate representation of the CFD data for both lift and drag coefficients. This may indicate that the trend of these coefficient is response 
to the variations in $l_{U}$ and $l_{L}$ is a more linear than a parabolic. In addition, despite their simplicity, second-order polynomial regression surrogates can provide a fairly-accurate representation of the data. This is more pronounced in the lift coefficient surrogate.

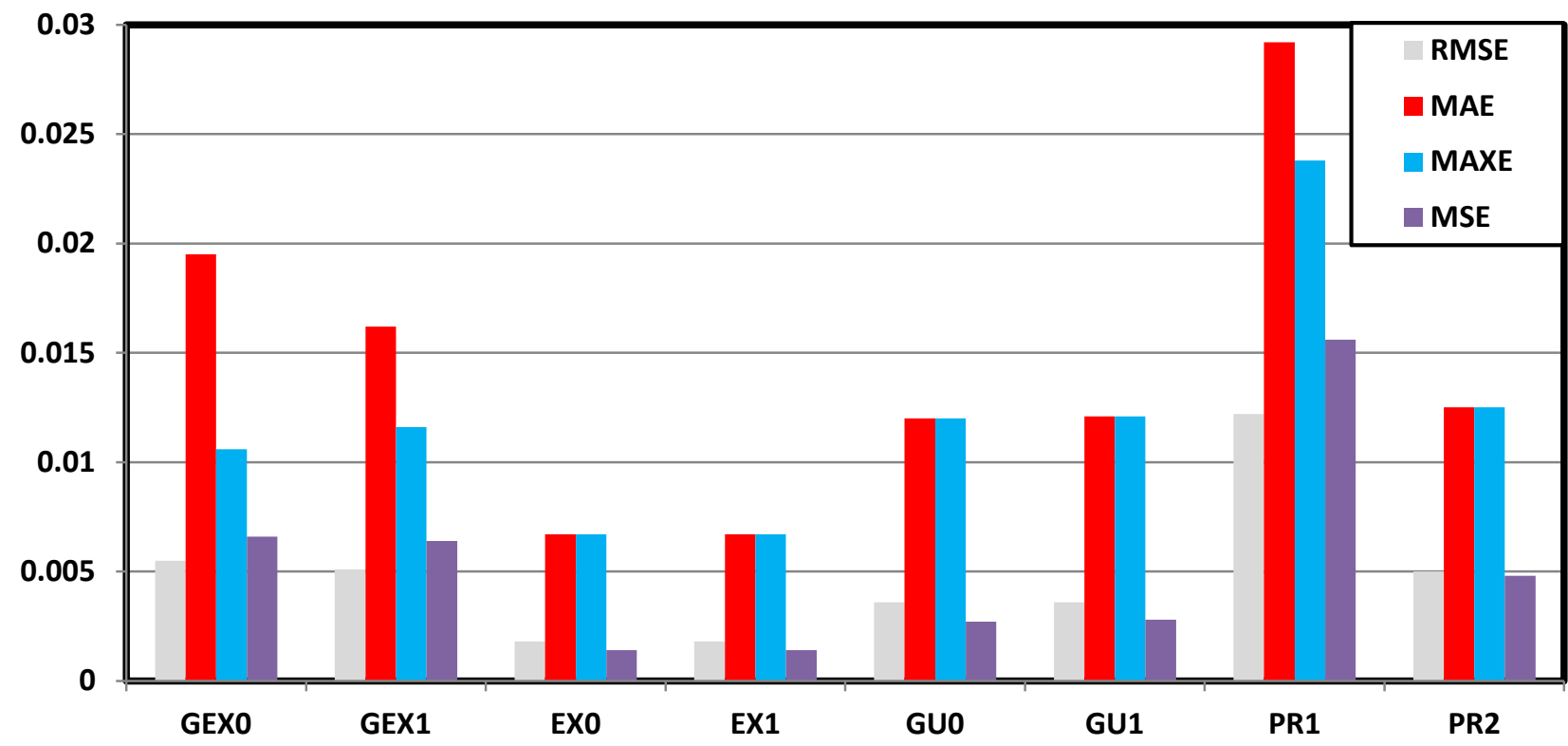

(a)

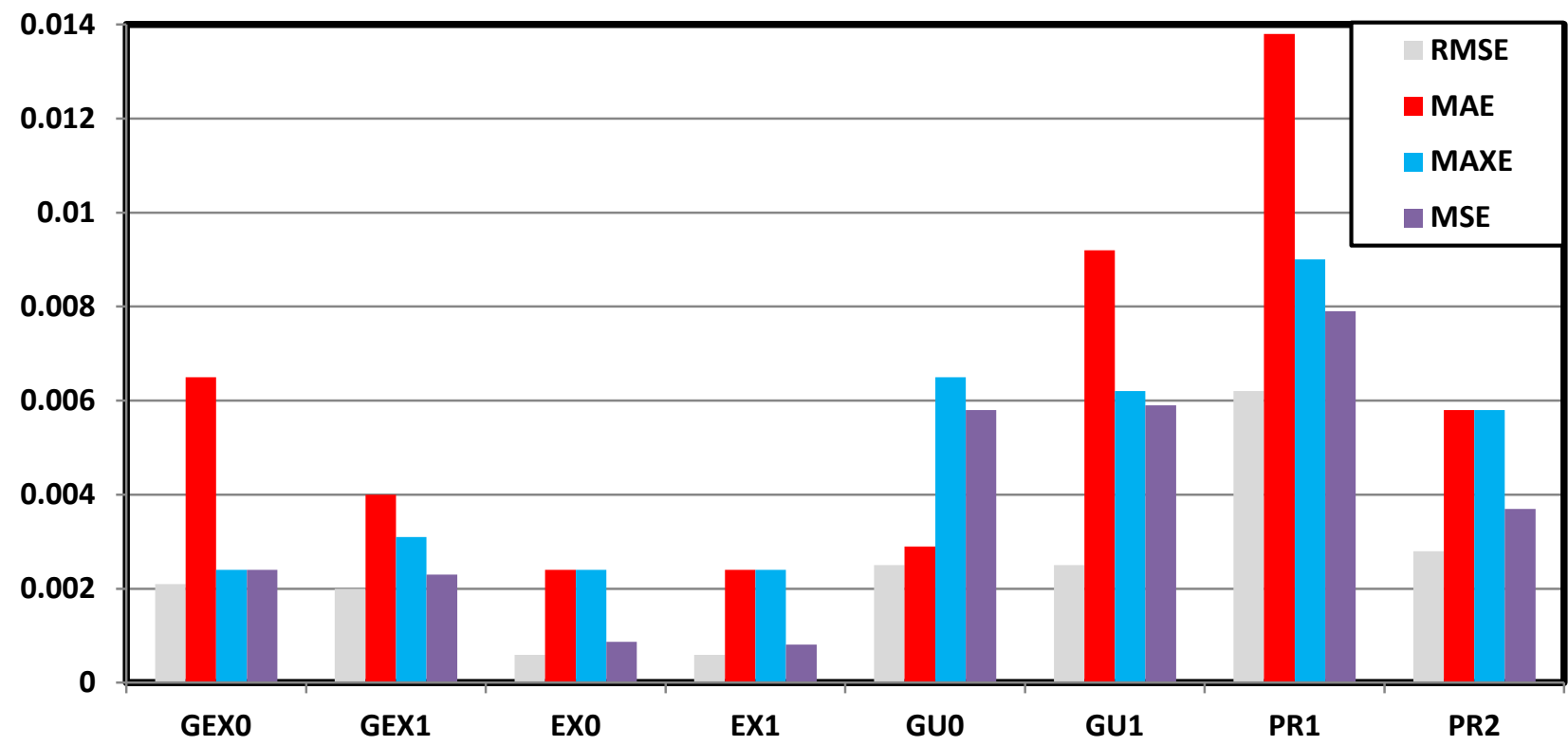

(b)

Figure 7 Measures of assessment for (a) lift coefficient surrogates, and (b) drag coefficient surrogates

\section{III.3. Representation and significance of the surrogates structures}

One significant feature of a surrogate is that it provides an explicit mathematical representation of the functional relation between parameters and response that can infer the underlying physics. In addition, a surrogate provides a visual (graphical) representation of the physical relation between parameters and response. These aspects are hard, sometimes impossible, to attain using solely the row data (numbers) originally used to construct the surrogate. These features are addressed in the present section. Focus is made on two surrogates that yield the highest accuracy namely, the exponential Kriging with first-order trend and the second-order polynomial regression. Here, all 104 airfoil designs are utilized to construct the surrogates i.e., training samples for the surrogates. 


\section{Lift coefficient surrogates}

The carpet curves shown Figure 8 are the graphical representation of the lift coefficient surrogates. The CFD values of the 104 samples used in surrogate construction are shown as solid dots.

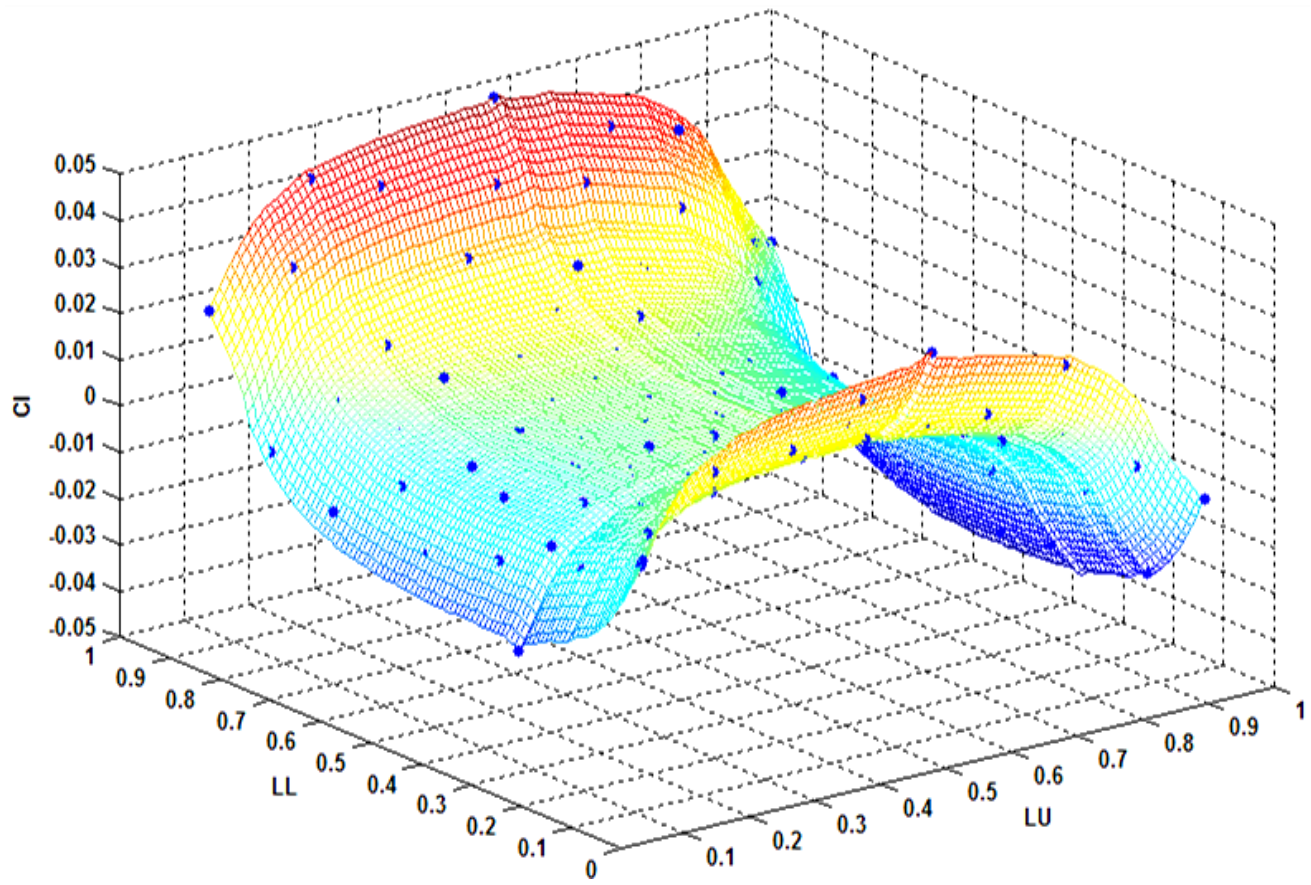

(a) Exponential Kriging with first-order trend

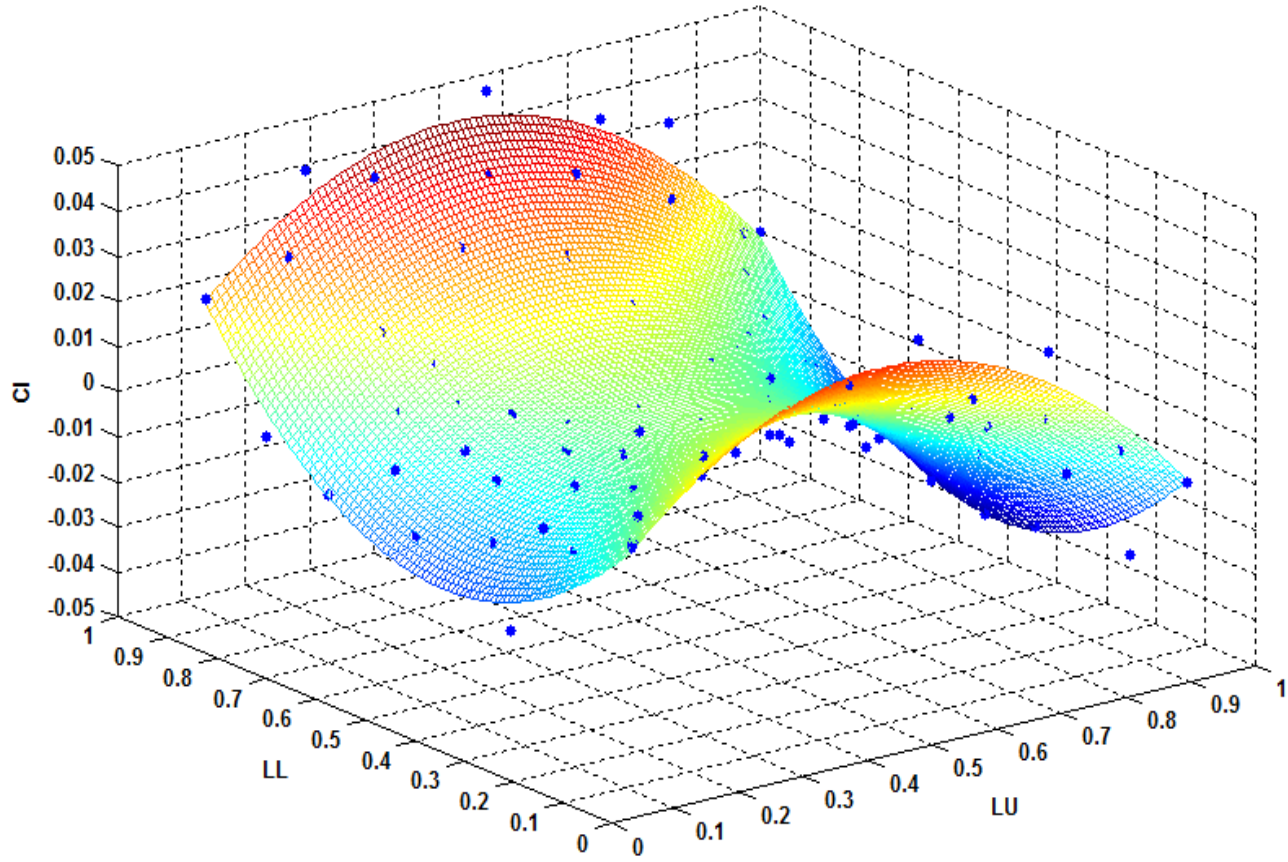

(b) Second-order polynomial regression Figure 8 Graphical representation of the lift surrogates

From the graphical form of the surrogate, the response surface, the relations between design parameters and output can be inferred. For instance, it is clear that lift is maximized at extreme (both low and high) values of $l_{L}$; intermediate values reduce the lift. In contrast, extreme values of $l_{U}$ reduce the lift while intermediate values increase it.

Since the freestream is at zero incidence, airfoil samples can yield positive and negative lift coefficients. Moreover, the interpolation nature of the Kriging surrogate and the 
regression nature of the polynomial surrogate are clear. While Kriging surrogate passes by all training samples, polynomial regression surrogate fits itself among them. This makes the polynomial surrogate smoother but less accurate. However, the significant advantage of polynomial regression surrogates is that they yield simple mathematical representation of the parameters-response relation. For instance, the mathematical formulation of PR2 lift surrogate is:

$c_{l}=-0.0017+0.1905 l_{U}-0.1834 l_{L}-0.182 l_{U} l_{L}-0.002 l_{U}^{2}+0.1776 l_{L}^{2}$

Despite its simplicity, a lot about the design-response relations for the airfoil lift coefficient can be inferred from the above formula. For instance:

- The positive value of $\beta_{1}(+0.1905)$ and the negative value of $\beta_{2}(-0.1834)$ indicate that the lift increases as $l_{U}$ increases and $l_{L}$ decreases. Physically, this is owed to the increase in shock wave strength and hence, the rise in flow pressure over the lower surface as $l_{L}$ decreases. The opposite is true for the shock wave ahead of the upper surface. The overall effect is the increase in lift coefficient.

- The absolute values of $\beta_{1}$ and $\beta_{2}$ are very close to each other. This indicates that $l_{U}$ and $l_{L}$ have almost the same level of dominance in the value of lift coefficient.

- Comparing the values of $\beta_{1}(+0.1905)$ and $\beta_{4}(-0.002)$ indicates that $l_{U}-c_{l}$ relation is more linear whereas the $l_{L}-c_{l}$ relation is more nonlinear.

\section{Drag coefficient surrogates}

Similarly, the graphical representations of the drag coefficient surrogates are shown in Fig. 9. Drag reaches maximum values for very small values of $l_{U}$ and $l_{L}$. As $l_{U}$ and $l_{L}$ increase towards their intermediate values, drag decreases almost monotonically. It reaches its minimum value nearly at the middle of the design space which is interpreted as a nearlysymmetric airfoil. Drag then increases steeply as $l_{U}$ and $l_{L}$ increase towards their maximum values.

The mathematical expression of the second-order polynomial regression surrogate is:

$$
c_{d}=0.083-0.0943 l_{U}-0.0938 l_{L}+0.076 l_{U} l_{L}-0.001 l_{U}^{2}+0.0751 l_{L}^{2}
$$

Examining the above equation indicates that:

- $l_{U}$ and $l_{L}$ have almost the same level of dominance with respect to the drag coefficient.

- Drag acting on the airfoil increases as both $l_{U}$ and $l_{L}$ decrease and vice versa.

- Drag is relatively more linear with respect to $l_{L}$ is relatively more nonlinear with respect to $l_{U}$. This is also inferred from the form of the carpet curves in Fig. 9. 


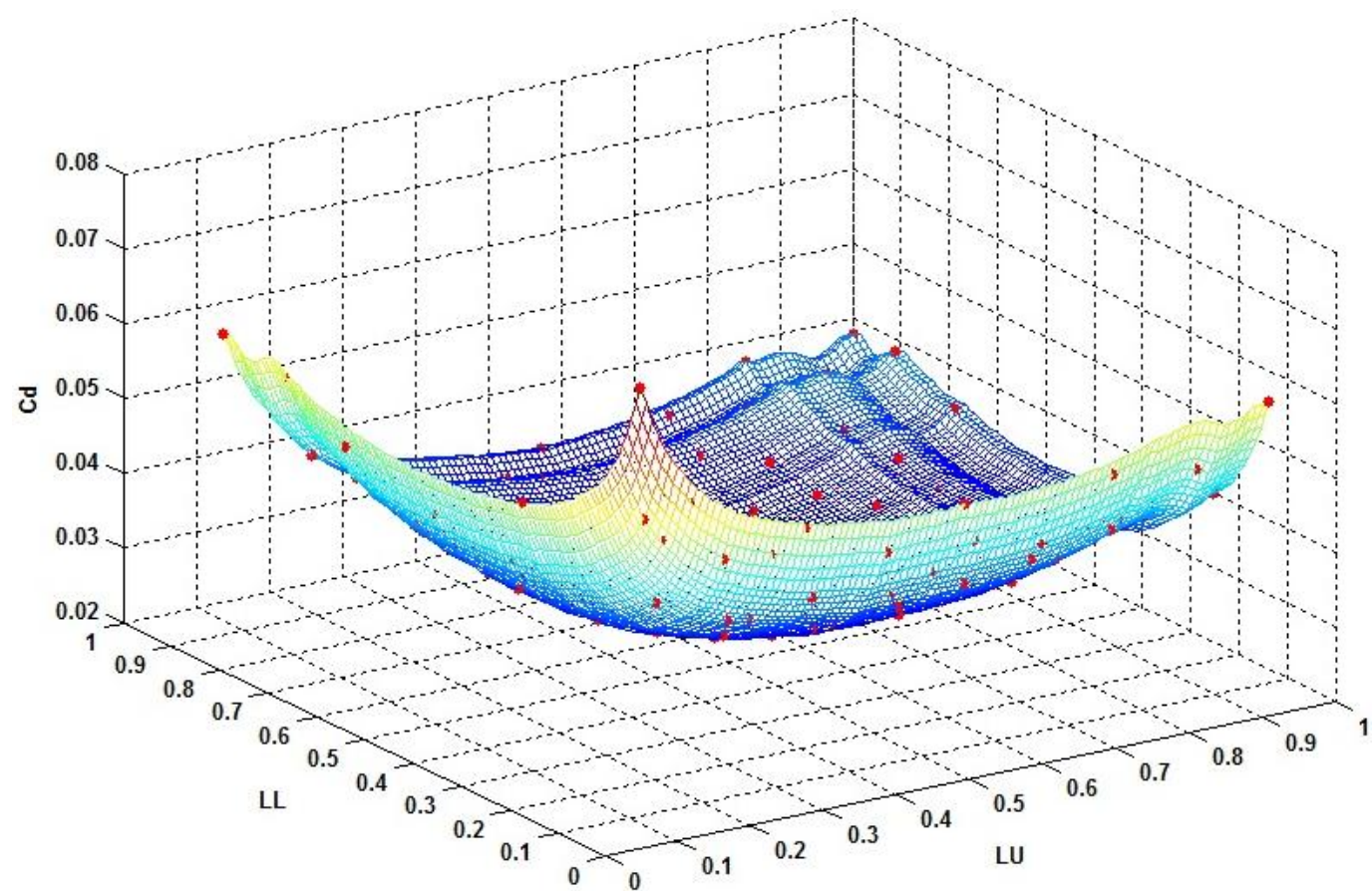

(a) Exponential Kriging with first-order trend

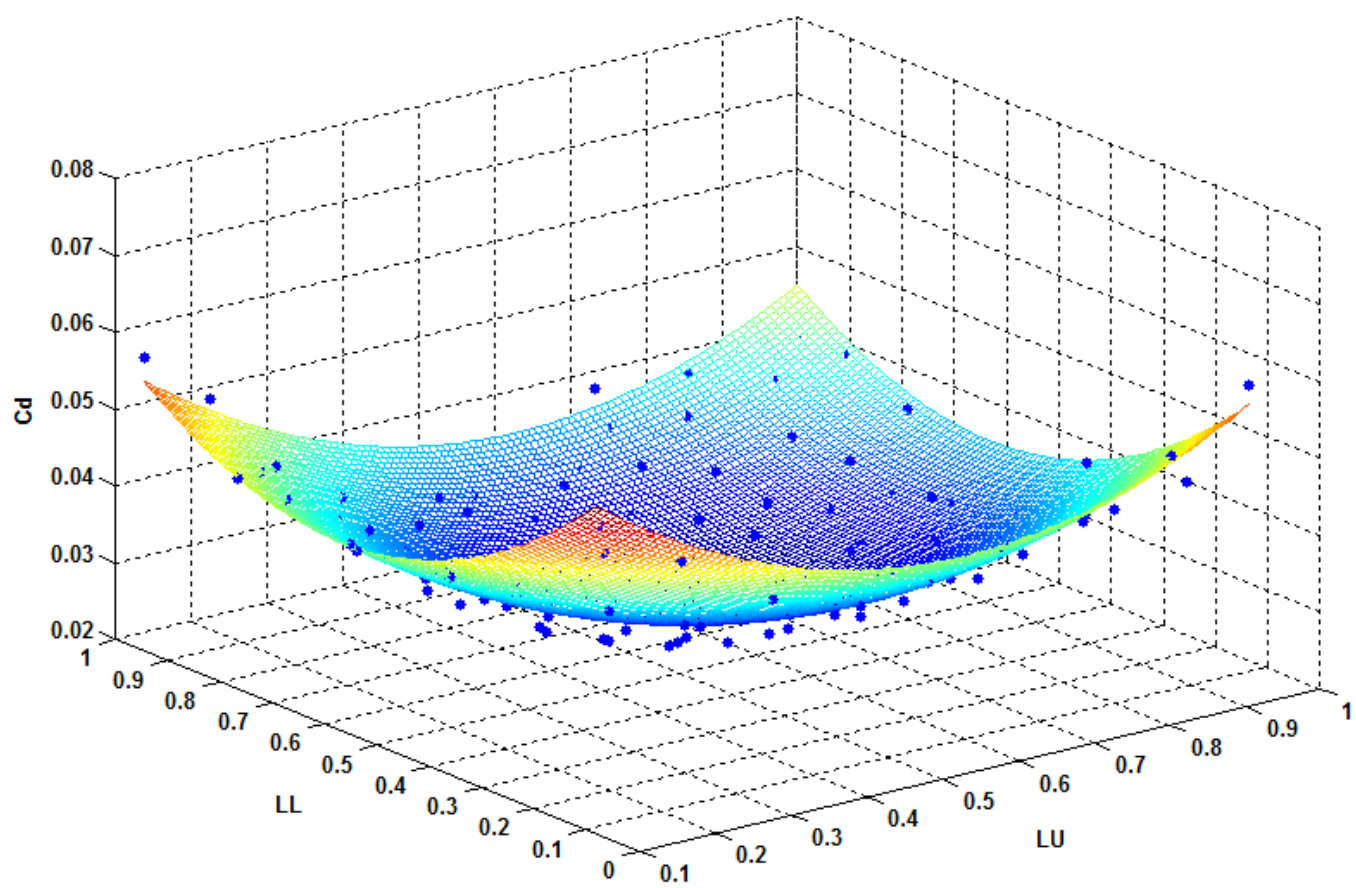

(b) Second-order polynomial regression

Figure 9 Graphical representation of the drag surrogates

\section{Conclusions}

Surrogates, based on data originally taken form lab or computer experiments, yield mathematical expressions and visual representations in lieu of theoretical equations. These alternative models have recently emerged, evolved, and been implemented in various applications. In the present paper, surrogate shave been used in a new application namely, dependence of diamond airfoil aerodynamic coefficients in its geometry. Kriging and polynomial regression surrogates were constructed and assessed based on data taken from CFD simulations of the flow around airfoils of different designs. The concept was successfully validated and the features of surrogates were addressed. The same framework established in the paper can be applied to other freestream conditions and airfoil designs. 
Surrogates constructed in the present work can be further utilized in design optimization studies of the supersonic airfoils.

\section{References}

[1] Ivey, H. R., Stickle, G. W., and Schuettler, A., "Charts for Determining the Characteristics of Sharp-Nose Airfoils in Two-Dimensional Flow at Supersonic Speeds,". NACA TN $1143,1947$.

[2] Anderson, J. D., "Fundamentals of Aerodynamics, Third Edition," McGraw Hill, 2001.

[3] Montgomery, D. C. and Evans, D. M. Jr., "Second-order Response Surface Designs in Computer Simulation," Simulation, Vol. 25,1975, pp. 169-178

[4] Bhadra, S. and Ganguli, R., "Aeroelastic Optimization of a Helicopter Rotor Using Orthogonal Array-Based Metamodels," AIAA Journal, Vol. 44, No. 9, 2006, pp. 1941 1951

[5] Manokaran, K., Vidya, G., Sivaramakrishnan, A. E., and Goyal, V. K., "Wing Planform Design Optimization for Reusable Launch Vehicle," Journal of Aircraft, Vol. 46, No. 2, 2009, pp. 726-730

[6] Kim H. and Liou, M., "New Multi-Objective Genetic Algorithms for Diversity and Convergence Enhancement," AIAA paper 2009-1168, 2009

[7] Koch, P. N., Mavris, D., and Mistree, F., "Partitioned, Multilevel Response Surfaces for Modeling Complex Systems," AIAA Journal, Vol. 38, No. 5, 2000, pp. 875-881.

[8] Sevant, N. E., Bloor, M. I. G., and Wilson, M. J., " Aerodynamic Design of a Flying Wing Using Response Surface Methodology," Journal of Aircraft, Vol. 37, No. 4, 2000, pp. 562- 569.

[9] Vavalle, A. and Qin, N., "Iterative Response Surface Based Optimization Scheme for Transonic Airfoil Design," Journal of Aircraft, Vol. 44, No. 2, 2007, 365- 376

[10] Williams, R. J., Evans, T. P. and McParlin, S. C., "Estimation of the Aerodynamic Characteristics of Cranked Wings through Response Surface Modelling," AIAA paper 2009-1095, 2009

[11] D. G. Krige, "A study of gold and uranium distribution patterns in the Klerksdorp gold field," Geoexploration, vol. 4, no. 1, pp. 43-53, 1966.

[12] Sacks, J., Welch, W. J., Mitchell, T. J., and Wynn, H. P., "Design and Analysis of Computer Experiments," Statistical Science, Vol. 4, No. 4, 1989, pp. 409-423

[13] Martin, J. D. and Simpson, T. W., "Use of Kriging Models to Approximate Deterministic Computer Models," AIAA Journal, Vol. 43, No. 4, 2005, pp. 853-863

[14] Forrester, A. I. J., Keane, A. J., and Bressloff, N. W., "Design and Analysis of "Noisy" Computer Experiments," AIAA Journal, Vol. 44, No. 10, 2006, pp. 2331-2339

[15] Kanazaki, M., Tanaka, K., Jeong, S., Yamamoto, K. "Multi-Objective Aerodynamic Exploration of Elements' Setting for High-Lift Airfoil Using Kriging Model," Journal of Aircraft, Vol. 44, No. 3, 2007, pp.858-864

[16] Meunier, M., "Simulation and Optimization of Flow Control Strategies for Novel HighLift Configurations," AIAA Journal, Vol. 47, No. 5, 2009, pp. 1145-1157

[17] Song, W. and Keane, A. J., "Surrogate-Based Aerodynamic Shape Optimization of a Civil Aircraft Engine Nacelle," AIAA Journal, Vol. 45, No. 10, 2007, pp. 2565-2574

[18] Ahmed, M. Y. M. and Qin, N., "Surrogate-Based Multi-Objective Aerothermodynamic Design Optimization of Hypersonic Spiked Bodies," AIAA Journal, 2012, Vol.50, pp: 797-810.

[19] Wintzer, M., Sturdza, P., and Kroo, I., "Conceptual Design of Conventional and Oblique Wing Configurations for Small Supersonic Aircraft," AIAA paper 2006-930, 2006

[20] Lisk, D., Robinson, T., and Robinson, D., "Multi-Objective Optimization of Supersonic Projectiles using Evolutionary Algorithms," AIAA paper 2010- 1500, 2010 
[21] McKay, M. D., Beckman, R. J., Conover, W. J., "A Comparison of Three Methods for Selecting Values of Input Variables in the Analysis of Output from a Computer Code," Technometrics, Vol. 21, No. 2, 1979, pp. 239-245

[22] Meckesheimer, M., Booker, A. J., Barton, R. R., and Simpson, T. W., "Computationally Inexpensive Metamodel Assessment Strategies," AIAA Journal, Vol. 40, No. 10, 2002, pp.2053-2060

[23] Queipo, N. V., Haftka, R. T., Shyy, W., Goel, T., Vaidyanathan, R., and Tucker, K., "Surrogate-based Analysis and Optimization," Progress in Aerospace Sciences, Vol. 41, 2005, pp. 1-28 